\title{
Ten kilodalton heat shock protein (HSP10) is overexpressed during carcinogenesis of large bowel and uterine exocervix
}

\author{
Francesco Cappello*, Marianna Bellafiore, Sabrina David, \\ Rita Anzalone, Giovanni Zummo \\ Department of Experimental Medicine, University of Palermo, via alla Falconara 120, Palermo 90136, Italy
}

Received 22 November 2002; received in revised form 21 February 2003; accepted 27 February 2003

\begin{abstract}
In the present study, we evaluated the presence and the level of expression of HSP10 in two carcinogenetic models: the 'adenoma-carcinoma sequence' of large bowel and the 'dysplasia-carcinoma sequence' of uterine exocervix. We found HSP10 was overexpressed during the carcinogenesis of both organs. In particular, HSP10 was overexpressed early in large bowel carcinogenesis, while the expression of this protein in exocervical carcinogenesis gradually increased from normal through dysplastic to neoplastic tissues. The quantitative analysis of immunohistochemistry and the Western blotting confirmed these results. Our previous observations showed overexpression of HSP60 in the same carcinogenetic models. This report correlates the overexpression of HSP10 with that of HSP60 during carcinogenesis in vivo. These results could stimulate further studies on the pathogenetic role of these proteins during the carcinogenesis as well as their use as diagnostic and prognostic tools in oncology.
\end{abstract}

(C) 2003 Elsevier Science Ltd. All rights reserved.

Keywords: Heat shock proteins; Carcinogenesis; Dysplasia; Carcinoma

\section{Introduction}

Heat shock protein (HSP) 10 is a $10 \mathrm{kDa}$ mitochondrial chaperone involved in protein folding. It has been highly conserved during phylogenesis and its function is strictly related to another mitochondrial chaperone, the HSP60, in both prokaryotic and eukaryotic cells [1]. The HSPs are involved in many cellular responses, including oxidative stress, and

\footnotetext{
* Corresponding author. Tel./fax: + 39-091-6553518.

E-mail address: francapp@hotmail.com (F. Cappello).
}

their role has been recently postulated also in cancer development. However, only a few works have discussed the possible role of HSP10 during carcinogenesis [1,2], while the involvement of HSP60 has been investigated by a number of studies [3-9]. We have recently showed an up-regulation of HSP60 during carcinogenesis of the large bowel [10] and the uterine exocervix [11]. We proposed that the overexpression of HSP60 could have a role during tumorigenesis, but these data need further support.

In the present study, we described the presence and the level of expression of HSP10 during colorectal 
and exocervical carcinogenesis, to add new knowledge on the role of these mitochondrial chaperones during the development of cancer.

\section{Materials and methods}

\subsection{Specimen collection}

In order to study the large bowel carcinogenesis, we collected 15 specimens from normal colonic mucosa (NC), 15 from tubular adenomas with mild dysplasia (MA), 15 from tubular adenomas with severe dysplasia (SA), and 15 from colorectal adenocarcinomas with an intermediate grade of differentiation (CA).

In order to study exocervical carcinogenesis, we collected 20 biopsies from normal exocervices (NE), 20 from low-grade squamous intraepithelial lesions (L-SIL), 20 from high-grade squamous intraepithelial lesions (H-SIL), and 20 from squamous carcinomas with an intermediate grade of differentiation (SC).

A small specimen of fresh tissue from each biopsy was frozen for Western blotting analysis. The rest of the biopsy was formalin-fixed and paraffin-embedded for pathologic diagnosis and immunohistochemical study.

\subsection{Immunohistochemistry}

Immunostaining by a streptavidin-biotin complex method (LSAB2 kit peroxidase, DAKO Corporation, Carpinteria CA, Cat. No. K0677) was performed using a primary antibody against HSP10 (Rabbit AntiCpn10 Polyclonal Antibody, StressGen, Biotechnologies Corp., Victoria, BC, Canada, Cat. No. SPA-110) at the dilution of 1:400 on 5-micra formalin-fixed paraffin-embedded sections. After incubation for 10 min with protein block serum-free (DAKO Corporation, Cat. No. X0909), the primary antibody was added to the sections. Non-immune rabbit serum was substituted for negative controls. Diaminobenzidine was used as develop chromogen (DAKO Corporation, Cat. No. K3467). Hematoxylin aqueous formula (DAKO Corporation, Cat. No. S2020) was used as counterstaining. The results were assessed by two independent observers.

\subsection{Quantitative analysis}

The expression of HSP10 in the examined specimens was quantified by computer-assisted image analysis (Colourvision 1.7.6, Improvision, Coventry, UK). For each biopsy, the tissue was systematically assessed in two non-serial sections, on the basis of red, green and blue (RGB) color balance. At the beginning of each session, the image analysis system was standardized using the same section of tissue stained for the HSP10 to ensure reproducibility of analysis. The digitized image of the standard section was used to sample interactively an example of the positive staining and the system was then allowed to select all the pixels of the same RGB color balance (i.e. positive staining) within the image. The area of the examined tissue was then delineated interactively and the percentage of positive staining within the tissue was determined; the color balance and percentual staining value was recorded for future sessions. At the beginning of each subsequent session, the image analyzer was calibrated using this section and adjusted to within $\pm 5 \%$ of the original pixel reading. Once the system had been calibrated using the 'standard' slide, the test sections were analyzed using the same parameters. An observer performed measurements of HSP10 expression. Each tissue section was analyzed on two separate occasions by two independent observers; means of the duplicate observations data were analyzed using Mann-Whitney $U$ test $P<0.05$ was considered significant.

\subsection{Western blot analysis}

Twenty micrograms of total cell extracts were applied to each lane along with a protein marker (Kaleidoscope prestained standard, Bio-Rad, Hercules, CA, USA, Cat. No. 1610324). They were separated by electrophoresis on denaturing $10 \%$ polyacrylamide slab gel (sodium dodecyl sulfatepolyacrylamide gel electrophoresis (SDS-PAGE)) and transferred to nitrocellulose membrane (Nitrocell Paper, Bio-Rad, Cat. No. 1620115). After $1 \mathrm{~h}$ at room temperature (RT) with a blocking buffer (5\% low-fat dried milk in Tampone Buffered Saline (TBS): $50 \mathrm{mM}$ Tris- $\mathrm{HCl}$ pH 7.5, $150 \mathrm{mM} \mathrm{NaCl}, 0.1 \%$ Tween-20) under gentle shaking, the membranes were incubated with anti-HSP10 primary antibody 
(Rabbit Anti-Cpn10 Polyclonal Antibody, StressGen, Biotechnologies Corp., Cat. No. SPA-110) at the dilution of 1:1000, overnight at $4^{\circ} \mathrm{C}$. After washing, the membranes were incubated with horseradish peroxidase (HRP)-conjugated secondary antibody (anti-rabbit, Pierce, 1:10 000, Cheshire, UK, Cat. No. 31432) for $1 \mathrm{~h}$ at RT with shaking and the specific binding was detected using a chemiluminescent substrate (SuperSignal West Pico Chemiluminescent Substrate, Pierce, Cat. No. 34080) for autoradiography.

\section{Results}

NC showed tubular glands of regular aspect with scattered inflammatory cells in lamina propria (Fig. 1a). Few cellular elements showed a punctate staining pattern for HSP10 [quantitative analysis: percent epithelial staining (range): $6(2-14)]$, consistent with a mitochondrial localization, while connective cells of lamina propria were negative (Fig. 1a, inset).

MA showed enlarged glands with cell and nuclear crowding. Inflammatory cells in lamina propria were abundant (Fig. 1b). Dysplastic glands showed a great number of positive cells [68 (53-81)], while lamina propria was negative (Fig. 1b, inset).

SA showed elongated and distorted glands with interposed abundant inflammatory stroma (Fig. 1c). Cellular elements in dysplastic glands were strongly positive [72 (56-90)]. Lamina propria was negative or, only occasionally, slightly positive in a few cells.

CA showed a cribrous architecture of tumoral glands; a great number of cells (Fig. 1d) had a diffuse cytoplasmic staining for HSP10 [77 (60-95)], while lamina propria showed only scattered positive cells.

The statistical evaluation of quantitative analysis showed that there was no significant difference between the levels of expression in MA, SA and $\mathrm{CA}$, but there was a significant increase in expression in all three conditions compared with $\mathrm{NC}$, as determined by both immunohistochemistry and immunoblotting. (Figs. 2a and 3a).

NE showed a multi-layered epithelium with scattered positive cells in basal layer (Fig. 1e). Parabasal, intermediate and superficial layers showed negative cellular elements (Fig. 1e, inset). The quantitative analysis showed that the percentage of epithelial staining was [8 (4-16)]. Lamina propria was negative.

L-SIL showed a mild thickening of epithelium and an inflammatory stroma (Fig. 1f). We found the presence of HSP10 in the cells of basal, parabasal and intermediate layer [433 (27-64)] (Fig. 1f, inset). Few scattered positive cells were also found in lamina propria.

H-SIL showed numerous positive cellular elements in the thickened epithelium (Fig. 1g). These cells were present in all layers of epithelium (Fig. 1g, inset) [62 (39-75)], while lamina propria showed rare elements staining for HSP10.

SC were characterized by islets of neoplastic cells with a positive staining (Fig. 1h). A great number of tumoral cells stained for HSP10 (Fig. 1h, inset) [84 (73-96)], while the reactive stroma showed scattered positive elements.

The statistical evaluation of quantitative analysis showed the presence of significant differences between the expression of HSP10 in NE, L-SIL, H-SIL and SC (Fig. 2b). Moreover, Western blot showed also a growing expression of HSP10 from NE through SILs to carcinomas (Fig. 3b).

\section{Discussion}

Heat shock proteins are a highly conserved family of molecules involved in protein folding in both prokaryotic and eukaryotic cells [12]. In particular, HSP10, also known as Cpn10, is the GroES Escherichia coli homologous protein present in mammal mitochondria [13]. HSP10 and HSP60 strictly interact in a two-step folding mechanism in normal prokaryotic and eukaryotic cells [14].

The role of HSPs during carcinogenesis was postulated and investigated in vivo in many sites, as lung [15], breast [16], esophageal [17] and ovarian cancer [18], melanoma [19], lymphoblastic leukemia and nephroblastoma [20]. In particular, HSP60 involvement in cancer development [7,9-11,21,22], diagnosis and prognosis [3-6,8] addressed more studies than did HSP10.

We have recently described the overexpression of HSP60 in two carcinogenetic models, the 'adenomacarcinoma sequence' of large bowel [10] and the 'dysplasia-carcinoma sequence' of uterine exocervix 
Large bowel
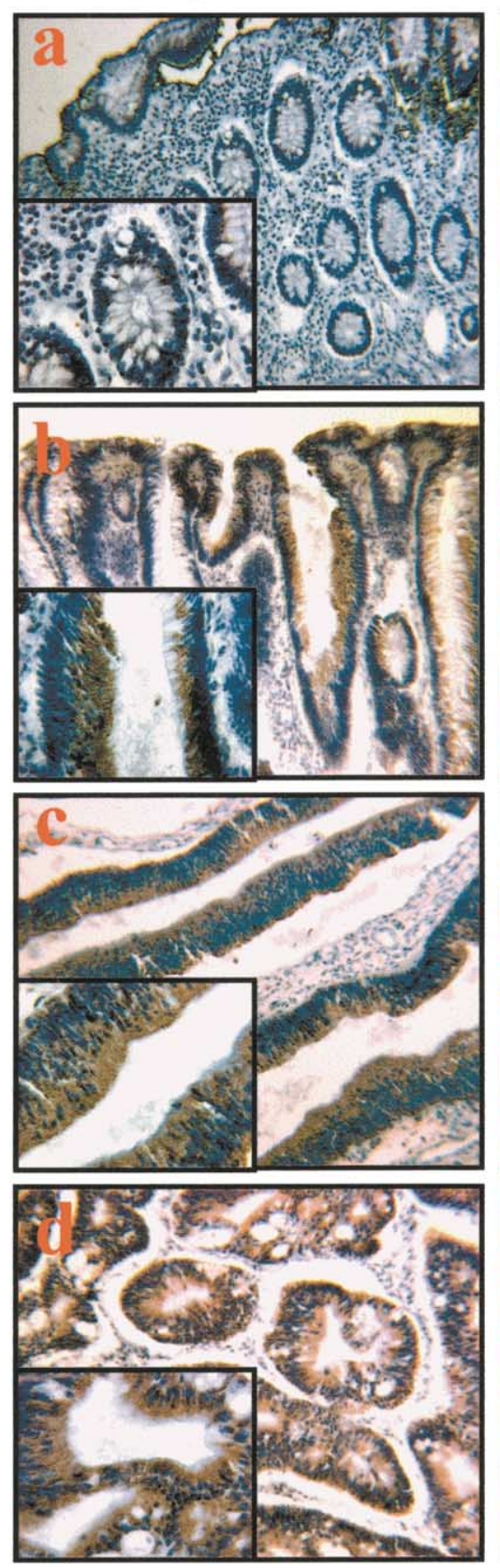

Exocervix
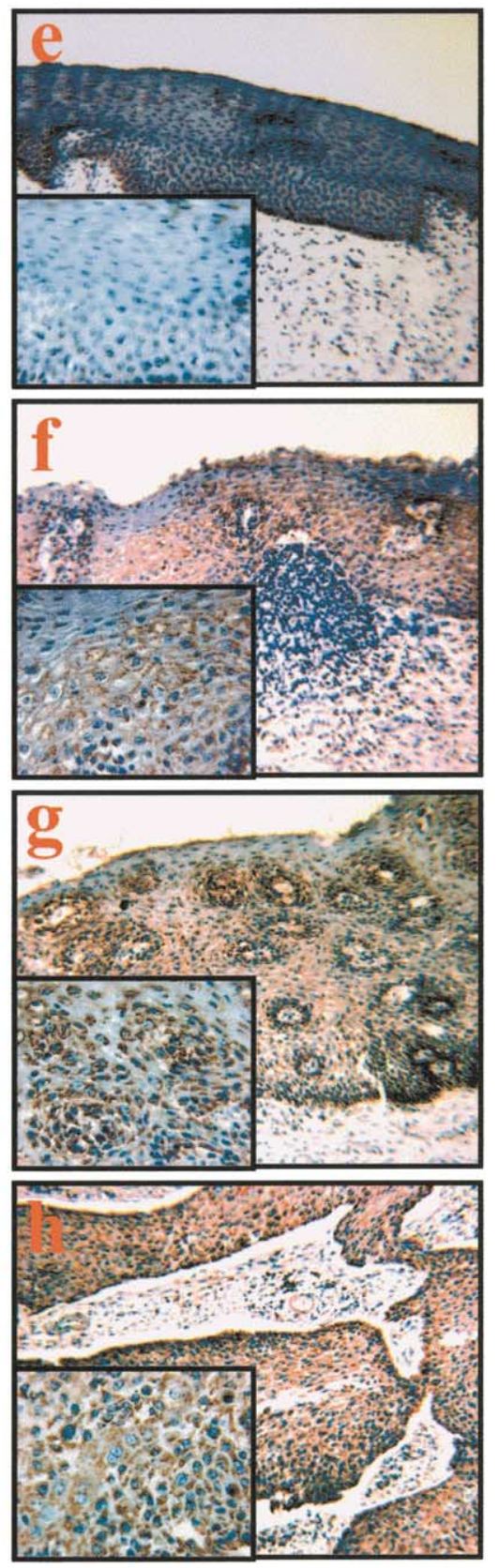

Fig. 1. Immunohistochemical positivity of HSP10 in normal colonic mucosa (a), tubular adenomas with mild dysplasia (b), tubular adenomas with severe dysplasia (c), colorectal adenocarcinomas with an intermediate grade of differentiation (d), normal exocervices (e), low-grade squamous intraepithelial lesions (f), high-grade squamous intraepithelial lesions (g) and squamous carcinomas with an intermediate grade of differentiation $(\mathrm{h})$ at $10 \times$ magnifications (inset: $40 \times$ ). 
a

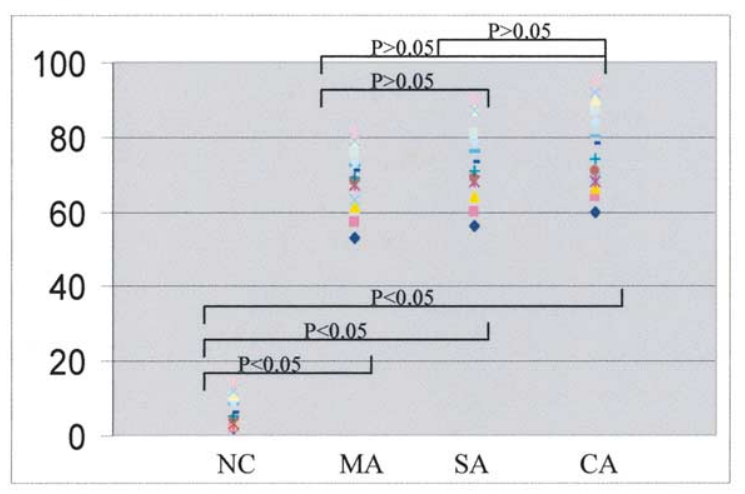

b

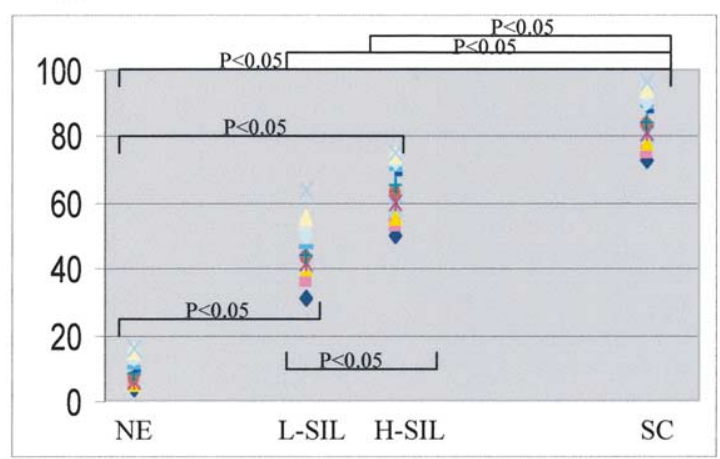

Fig. 2. (a) Statistic evaluation in large bowel carcinogenesis: NC differed in significant manner by MA, SA and CA; absence of significant differences among the positivity of MA, SA and CA. (b) Statistic evaluation in exocervical carcinogenesis: presence of significant differences among the positivity of NE, L-SIL, H-SIL and SC.

[11]. HSP60 cytoplasmic accumulation increased during exocervical carcinogenesis [11], while HSP60 accumulates early in cytoplasm of tubular adenomas of large bowel [10]. We proposed that HSP60 could have an active role in the multi-step process of carcinogenesis, i.e. during both promotion and progression steps. In the present study, we evaluated the presence and the level of expression of HSP10 in normal, dysplastic and neoplastic tissues obtained from human large bowels and uterine exocervices.

Immunohistochemical analysis showed positive staining for HSP10 in normal, dysplastic and neoplastic tissues in both large bowel and uterine exocervix. It was strongest in epithelial cells with only a few scattered cells in the connective tissue. The strength of positive staining increased from normal to carcinoma, as confirmed by quantitative and molecular analyses. In addition, we found a gradual accumulation of HSP10 in the 'dysplasia-carcinoma sequence' of uterine exocervix, while it was overexpressed early in cells of glands of MA. These data were also confirmed by both quantitative and molecular analyses.

In the present series of colonic tissues we obtained similar results to those of Somodevilla-Torres et al. [23]; they report that normal colonic tissue showed a punctate staining pattern for HSP10, consistent with a mitochondrial localization, while malignant bowel shows an overall increase in staining with diffuse cytoplasmic staining and localization in secretory structures and malignant gland spaces.

Rat models of cerebral [24,25] and cardiac ischemia [26,27] showed a simultaneous overexpression of HSP10 and HSP60. More recently, an up-regulation of these chaperones was demonstrated also in human myocardium during chronic atrial fibrillation [28]. These data indicate a protective role of these HSPs during the hypoxic-ischemic stress. In the present study, the data concerning the HSP10 expression during colorectal and exocervical

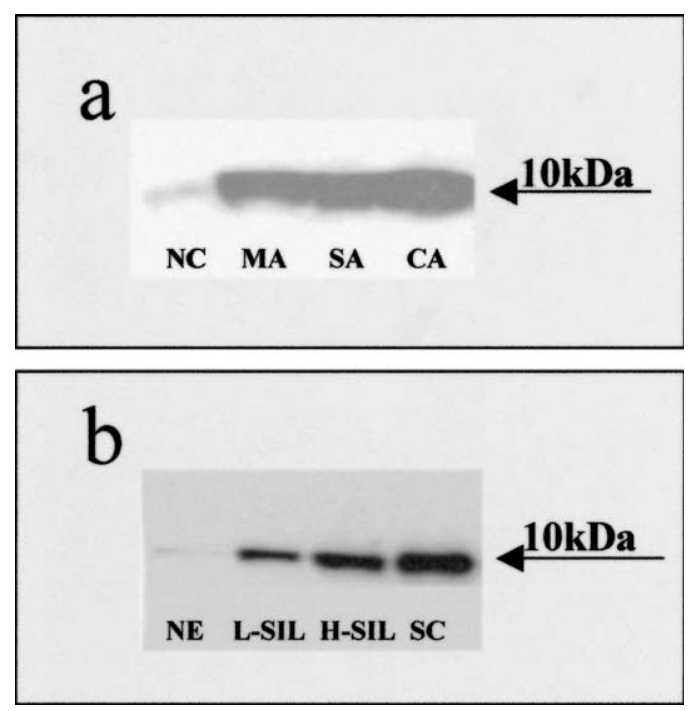

Fig. 3. Representative Western blot analyses in large bowel (a) and exocervical (b) carcinogenesis. 
carcinogenesis are similar to those we found for HSP60 $[10,11]$. This analogous pattern of overexpression could indicate that activities of HSP10 and HSP60 are both conserved. As a consequence, this overexpression could be a protective up-regulation and these HSPs could have a role in refolding of proteins that are denatured during carcinogenesis, as BRCA1 in breast cancer [29] and p16 in many other tumors [30].

Samali et al. [31] recently described a preapoptotic complex among pro-caspase 3, HSP60 and HSP10 in mitochondria of Jurkat cells. Induction of apoptosis led to the activation of pro-caspase 3 and its dissociation from the HSPs, which were released from mitochondria. This mechanism accelerated caspaseactivation in the cytoplasm of intact cells and promoted the trigger of apoptotic mechanism. Since defective apoptosis can induce tumorigenesis [32] during carcinogenesis, HSP10 and HSP60 could lose their pro-apoptotic role and these defective proteins could accumulate in cytoplasm of dysplastic and/or neoplastic cells. The two proposed mechanisms (protective up-regulation or defective accumulation) are not mutually exclusive, but could represent a continuum in the pathogenesis of tumors, from dysplasia to cancer.

In conclusion, to our knowledge, this should be the first study to correlate the simultaneous overexpression of HSP10 and HSP60 during carcinogenesis. We believe that these proteins could be functional in the cytoplasm of both dysplastic and neoplastic cells, but this hypothesis needs further confirmation. Our data may stimulate further pathogenetic studies to understand better why these chaperones are overexpressed during carcinogenesis. Finally, these data could have also a clinical relevance because HSPs could become new diagnostic and prognostic tools for the management of neoplasms.

\section{Acknowledgements}

This work was funded by 'M.I.U.R. ex-60\%' and 'Progetto Giovani Ricercatori'. We are grateful to Dr Marcianò for technical support.

\section{References}

[1] A.C. Cavanagh, H. Morton, The purification of earlypregnancy factor to homogeneity from human platelets and identification as chaperonin 10, Eur. J. Biochem. 222 (1994) $551-560$.

[2] G. Galli, P. Ghezzi, P. Mascagni, F. Marcucci, M. Fratelli, Mycobacterium tuberculosis heat shock protein 10 increases both proliferation and death in mouse P19 teratocercinoma cells, In Vitro Cell. Dev. Biol. Anim. 32 (1996) 446-450.

[3] K. Trieb, R. Gerth, R. Windhager, J.S. Grohs, G. Holzer, P. Berger, et al., Serum antibodies against the heat shock protein 60 are elevated in patients with osteosarcoma, Immunobiology 201 (2000) 368-376.

[4] P. Piselli, S. Vendetti, D. Vismara, R. Cicconi, F. Poccia, V. Colizzi, et al., Different expression of CD44, ICAM-1 and HSP60 on primary tumor and metastases of a human pancreatic carcinoma growing in scid mice, Anticancer Res. 20 (2000) 825-831.

[5] H. Uozaki, T. Ishida, C. Kakiuchi, H. Horiuchi, T. Gotoh, T. Iijima, et al., Expression of heat shock protein in osteosarcoma and its relationship with prognosis, Pathol. Res. Pract. 196 (2000) 665-673.

[6] P.A. Cornford, A.R. Dodson, K.F. Parson, A.D. Desmond, A. Woolfenden, M. Fordham, et al., Heat shock protein expression independently predicts clinical outcome in prostate cancer, Cancer Res. 60 (2000) 7099-7105.

[7] M. Castelli, F. Cianfriglia, A. Manieri, L. Palma, R.W. Pezzuto, A. Falasca, et al., Anti-p53 and anti-heat shock proteins antibodies in patients with malignant or premalignant lesions of the oral cavity, Anticancer Res. 21 (2001) 753-758.

[8] E. Ishii, K. Yokota, T. Sugiyama, Y. Fujinaga, K. Ayada, I. Hokari, et al., Immunoglobulin G1 antibody response to Helicobacter pylorii heat shock protein 60 is closely associated with low-grade gastric mucosa-associated lymphoid tissue lymphoma, Clin. Diagn. Lab. Immunol. 8 (2001) 1056-1059.

[9] H.O. Barazi, L. Zhou, N.S. Templeton, H.C. Krutzsch, D.D. Roberts, Identification of heat shock protein 60 as a molecular mediator of alpha 3 beta 1 integrin activation, Cancer Res. 62 (2002) 1541-1548.

[10] F. Cappello, M. Bellafiore, A. Palma, S. David, V. Marcianò, T. Bartolotta, et al., $60 \mathrm{kDa}$ Chaperonin (hsp60) is overexpressed during colorectal carcinogenesis, Eur. J. Histochem. 47 (2003) 105-109.

[11] F. Cappello, M. Bellafiore, A. Palma, V. Marcianò, G. Martorana, P. Belfiore, et al., $60 \mathrm{kDa}$ Heat shock protein (HSP60) expression increases during carcinogenesis of uterine exocervix, Pathobiology 70 (2002) 83-88.

[12] M.T. Ryan, D.J. Naylor, P.B. Hoj, M.S. Clark, N.J. Hoogenraad, The role of molecular chaperones in mitochondrial protein import and folding, Int. Rev. Cytol. 174 (1997) $127-193$.

[13] J. Hohfeld, F.U. Hartl, Role of the chaperonin cofactor HSP10 in protein folding and sorting in yeast mitochondria, J. Cell Biol. 126 (1994) 305-315.

[14] J.S. Weissman, C.M. Hohl, O. Kovalenko, Y. Kashi, S. Chen, 
K. Braig, et al., Mechanism of GroEL action: productive release of polypeptide from sequestred position under GroES, Cell 83 (1995) 577-587.

[15] R. Koomagi, G. Stammler, C. Manegold, J. Mattern, M. Volm, Expression of resistance-related proteins in tumoral and peritumoral tissues of patients with lung cancer, Cancer Lett. 110 (1996) 129-136.

[16] M. Yano, Z. Naito, M. Yokoyama, Y. Shiraki, T. Ishiwata M. Inokuchi, et al., Expression of hsp90 and cyclin D1 in human breast cancer, Cancer Lett. 137 (1999) 45-51.

[17] M. Nakajima, H. Kuwano, T. Miyazaki, N. Masuda, H. Kato, Significant correlation between expression of heat shock proteins 27, 70 and lymphocyte infiltration in esophageal squamous cell carcinoma, Cancer Lett. 178 (2002) 99-106.

[18] K. Yamamoto, A. Okamoto, S. Isonishi, K. Ochiai, Y. Ohtake, Heat shock protein 27 was up-regulated in cisplatin resistant human ovarian tumor cell line and associated with the cisplatin resistance, Cancer Lett. 168 (2001) 173-181.

[19] M.P. Protti, S. Heltai, M. Bellone, M. Ferrarini, A.A. Manfredi, C. Rugarli, Constitutive expression of the heat shock protein $72 \mathrm{kDa}$ in human melanoma cells, Cancer Lett. 85 (1994) 211-216.

[20] G. Stammler, M. Wolm, Expression of heat shock proteins, glutathione peroxidase and catalase in childhood acute lymphoblastic leukemia and nephroblastoma, Cancer Lett. 99 (1996) 35-42.

[21] E. Kimura, R.E. Enns, J.E. Alcaraz, J. Arboleda, D.J. Slamon, S.B. Howell, Correlation of the survival of ovarian cancer patients with mRNA expression of the 60-kd heat-shock protein HSP60, J. Clin. Oncol. 11 (1993) 891-898.

[22] T. Ito, R. Kawabe, Y. Kurasono, M. Hara, H. Kitamura, K. Fujita, et al., Expression of heat shock proteins in squamous cell carcinoma of the tongue: an immunohistochemical study, J. Oral Pathol. Med. 27 (1998) 18-22.

[23] M.J. Somodevilla-Torres, N.C. Hillyard, H. Morton, D. Alewood, J.A. Halliday, P.F. Alewood, et al., Preparation and characterization of polyclonal antibodies against human chaperonin 10, Cell Stress Chaperones 5 (2000) 14-20.

[24] A. Okubo, H. Kinouchi, Y. Owada, H. Kunizuka, H. Otoh, K. Izaki, et al., Simultaneous induction of mitochondrial heat shock protein mRNAs in rat forebrain ischemia, Brain Res. Mol. Brain Res. 84 (2000) 127-134.

[25] K. Izaki, H. Kinouchi, K. Watanabe, Y. Owaka, A. Okubo, H. Itoh, et al., Induction of mitochondrial heat shock protein 60 and 10 mRNAs following transient focal cerebral ischemia in the rat, Brain Res. Mol. Brain Res. 88 (2001) 14-25.

[26] S. Lau, N. Patnaik, M.R. Sayen, R. Mestril, Simultaneous overexpression of two stress protein in rat cardiomyocytes and myogenic cells confers protection against ischemia-induced injury, Circulation 96 (1997) 2287-2294.

[27] W.H. Dillmann, Heat shock proteins and protection against ischemic injury, Infect. Dis. Obstet. Gynecol. 7 (1999) 55-57.

[28] A.E. Schafler, K. Kirmanoglou, P. Pecher, A. Hannekum, B. Schumacher, Overexpression of heat shock protein $60 / 10$ in myocardium of patients with chronic atrial fibrillation, Ann. Thorac. Surg. 74 (2002) 767-770.

[29] C.M. Ekblad, H.R. Wilkinson, J.W. Schymkowitz, F. Rousseau, S.M. Freund, L.S. Itzhaki, Characterisation of the BRCT domains of the breast cancer susceptibility gene product BRCA1, J. Mol. Biol. 320 (2002) 431-442.

[30] K.S. Tang, B.J. Guralnick, W.K. Wang, A.R. Fersht, L.S. Itzhaki, Stability and folding of the tumour suppressor protein p16, J. Mol. Biol. 285 (1999) 1869-1886.

[31] A. Samali, J. Cai, B. Zhivotovsky, D.P. Jones, S. Orrenius, Presence of a pre-apoptotic complex of pro-caspase-3, Hsp60 and $\mathrm{Hsp} 10$ in the mitochondrial fraction of jurkat cells, EMBO J. 18 (1999) 2040-2048.

[32] F. Cappello, M. Bellafiore, A. Palma, F. Bucchieri, Defective apoptosis and tumorigenesis. Role of p53 mutation and Fas/FasL dysregulation, Eur. J. Histochem. 46 (2002) 199-208. 